\title{
Salt intake monitoring at a population level
}

\author{
Arnaud Chiolero ${ }^{1,2,3}$
}

\section{To the Editor:}

I have read with great interest the scientific statement of the British and Irish Hypertension Society on the importance of a valid assessment of salt intake [1]. While I agree that 24-h urine collections, if complete, are the best method to assess salt intake, one should not underestimate the value of urine spot samples-with an adequate equation-to monitor salt intake at a population level [2-4].

To avoid confusion and oversimplification on how to measure salt intake, we have to be explicit on why we want to measure salt intake. It is indeed key to distinguish three goals: (1) to estimate salt intake at the individual level, e.g., to identify patients with a high intake and to follow-up their intake throughout time; (2) to estimate salt intake at the group or population level, e.g., for the monitoring of salt intake in a given population; and (3) to estimate the health risk associated with salt intake. On the one hand, at an individual level, urinary spots are not a convincing method. However, at that level, nothing is really convincing. If we want to estimate salt intake at an individual level, multiple 24-h urine collections would be needed what is not feasible out of a research setting. Urinary spot samples are surely also not adequate for estimating the health risk associated with salt intake, as measurement errors lead to serious biases [1, 5].

On the other hand, urinary spot samples could allow estimating salt intake at a population level. A crucial

Arnaud Chiolero

achiolero@gmail.com

Institute of Primary Health Care (BIHAM), University of Bern, Bern, Switzerland

2 Population Health Laborator, University of Fribourg, Fribourg, Switzerland

3 Department of Epidemiology, Biostatistics and Occupational Health, McGill University, Montreal, QC, Canada element is the ability of the equation to transform a sodium concentration in an excretion estimate. Hence, studies in adults [2] and recently in children [3, 4] have shown that urinary spot samples with proper equations (e.g., Tanaka or Brown) can fairly estimate 24-h urine excretion. Salt intake is however largely overestimated with the Kawasaki equation [2-4, 7]. Further research is ongoing to improve equations and to evaluate if spot urine samples can measure change in salt intake over time [6]. One should also not forget that not willing to collect urine over $24 \mathrm{~h}$ and incomplete collections are major difficulties of 24-h urine collections for salt monitoring at a population level, as individuals who agree to collect and give a complete sample are surely not representative of the general population.

More broadly, we should keep in mind that what is needed is a pragmatic method to estimate usual salt intake at a population level. We face similar challenges with the assessment of blood pressure (BP) [8]. To estimate usual BP, multiple 24-h BP measurements are certainly better than a couple of $\mathrm{BP}$ readings at office visits; it does however not mean that office BP cannot be used to monitor mean BP level and hypertension control at a population level [9]. By analogy, while multiple 24-h urinary collections are certainly better for estimating usual salt intake, urinary spot samples with the proper equation could be sufficient for the monitoring at a population level.

Funding This work was funded in part by the Swiss Federal Food Safety and Veterinary Office (funding reference number 5.15.03).

\section{Compliance with ethical standards}

Conflict of interest The author declares that he has no conflict of interest.

Publisher's note: Springer Nature remains neutral with regard to jurisdictional claims in published maps and institutional affiliations. 


\section{References}

1. Cappuccio FP, Sever PS. The importance of a valid assessment of salt intake in individuals and populations. A scientific statement of the British and Irish Hypertension. Soc J Hum Hyper 2019;33:345-8.

2. Huang L, Crino M, Wu JH, Woodward M, Barzi F, Land MA, et al. Mean population salt intake estimated from 24-h urine samples and spot urine samples: a systematic review and meta-analysis. Int J Epidemiol. 2016;45:239-50.

3. Rios-Leyvraz M, Bovet P, Tabin R, Genin B, Russo M, Rossier MF, et al. Urine spot samples can be used to estimate 24-hour urinary sodium excretion in children. J Nutr 2018;148:1946-53.

4. Dong J, Yan Y, Fan H, Zhao X, Mi J. Accuracy validation of 8 equations to estimate 24-hour sodium by spot urine in young adolescents. Am J Hypertens. 2019;32:257-64.

5. Hutcheon JA, Chiolero A, Hanley JA. Random measurement error and regression dilution bias. BMJ 2010;340:c2289.
6. Huang L, Crino M, Wu JH, Woodward M, Land MA, McLean R, et al. Reliable quantification of the potential for equations based on spot urine samples to estimate population salt intake: protocol for a systematic review and meta-analysis. JMIR Res Protoc. 2016;5: e190.

7. He FJ, Campbell NRC, Ma Y, MacGregor GA, Cogswell ME, Cook NR. Errors in estimating usual sodium intake by the Kawasaki formula alter its relationship with mortality: implications for public health. Int J Epidemiol. 2018;47:1784-95.

8. Muntner P, Shimbo D, Carey RM, Charleston JB, Gaillard T, Misra $S$, et al. Measurement of blood pressure in humans: a scientific statement from the American Heart Association. Hypertension. 2019;73:e35-e66.

9. Wolf-Maier K, Cooper RS, Banegas JR, Giampaoli S, Hense $\mathrm{H}$, Joffres $\mathrm{M}$, et al. Hypertension and blood pressure levels in 6 European countries, Canada, and the US. JAMA. 2003; 289:2363-2369. 